\title{
MODELO DE TEMPERATURA PARA REACTORES ABIERTOS DE MICROALGAS
}

\author{
E. Rodríguez ${ }^{1}$, F.C. Acién ${ }^{2}$, J.L. Guzmán ${ }^{3}$, M. Berenguel ${ }^{3}$, A. Visioli ${ }^{4}$ \\ ${ }^{1}$ Department of Mechanical and Industrial Engineering, University of Brescia, Brescia, 25123, Italy; \\ \{e.rodriguezmiran,antonio.visioli\}@unibs.it \\ ${ }^{2}$ Dep. de Ingeniería, Universidad de Almería, CIESOL, 04120 Almería, España. facien@ual.es \\ ${ }^{3}$ Dep. de Informática, Universidad de Almería, 04120, CIESOL AlcemeiA3r, ía, España.\{joseluis.guzman,beren\}@ual.es
}

\section{Resumen}

En este artículo se presenta un modelo de temperatura para reactores abiertos de tipo raceway basado en un balance térmico a partir de variables fácilmente medibles del ambiente. El modelo permite estimar la temperatura con gran precisión, siguiendo la dinámica observada en la temperatura del reactor estudiado. Gracias a este tipo de modelos se pueden desarrollar modelos de microalgas más completos, que permitan representar fielmente la dinámica de crecimiento de las mismas.

Palabras clave: Modelo de temperatura, microalgas, reactor raceway.

\section{INTRODUCCIÓN}

Recientemente, la implantación de reactores de microalgas para la producción de biomasa se está expandiendo debido a las ventajas y los productos que se pueden obtener de su cultivo. Las microalgas permiten obtener productos de alto valor que se emplean en la industria química [4] o como alimento en diversas áreas, como las piscifactorías. Más aún, gracias a su habilidad para producir lípidos, el enfoque actual se centra en la producción de biocombustible [10]. Recientemente, el uso de agua residual como medio de cultivo está permitiendo el desarrollo de nuevas aplicaciones combinadas como el tratamiento y purificación del agua junto a la producción de biomasa en un único reactor, reduciendo los costes de operación y potenciando el uso de las microalgas para otras aplicaciones, como la producción de biocombustible u otros usos de interés. El desarrollo de este tipo de aplicaciones se lleva a cabo en reactores abiertos o raceway, menos costosos y fáciles de operar que los fotobioreactores cerrados tubulares, pero con el inconveniente de tener un menor rendimiento de producción y estar enfocados a cepas de microalgas de valor medio-bajo.

Durante el proceso biológico de las microalgas, las variables más importantes que afectan al crecimiento del cultivo son la temperatura, la radiación solar, el pH y el oxígeno disuelto [2]. El diseño de un reactor abierto determina las condiciones de incidencia de la radiación solar y temperatura. Por tanto, las variables a controlar son el $\mathrm{pH}$ y el oxígeno disuelto, siendo necesario mantenerlas en unos niveles de operación establecidos a pesar de los cambios en las perturbaciones, tales como la radiación solar [9].

Los modelos biológicos de microalgas pueden ayudar a estimar la productividad en los cultivos, así como estimar parámetros característicos de los mismos que puedan ser utilizados en los sistemas de control para maximizar la producción de biomasa $[2,9]$. Comúnmente, estos modelos no tienen en cuenta la temperatura del medio, siendo sin embargo, una variable muy importante que afecta a la productividad y salud de la cepa. Existen algunos estudios realizados sobre la productividad y la temperatura del medio en reactores abiertos [5]. Además, en [1] se presenta un modelo universal de temperatura para reactores abiertos, que hace uso de parámetros adimensionales para la transferencia de calor y fenómenos de evaporación. Por otro lado, en [6] se desarrolla un modelo dinámico para el cultivo de microalgas donde se incluye un modelo empírico de temperatura basado en balances térmicos de energía sugerido en [12]. Estos estudios demuestran la importancia de la influencia de la temperatura sobre las microalgas y la complejidad de estimar fielmente el valor de la misma. Combinar un modelo de temperatura con los modelos actuales de biomasa permitiría obtener una mayor precisión en cuanto a la representación del comportamiento de las microalgas. Gracias a ello, se podrían desarrollar mejores arquitecturas de control para producción de biomasa y las aplicaciones asociadas.

En este artículo, se presenta un modelo de temperatura simple, basado en una revisión de las relaciones empíricas definidas en [12], aplicado a un reactor abierto. La temperatura se calcula a partir de un balance térmico en el reactor, teniendo en cuenta una serie de variables del ambiente. Este modelo permite estimar la temperatura del medio en el reactor para unas determinadas condiciones ambientales. De esta forma, con el modelo se podrían estimar parámetros de interés, como el momento de cosechado o anticipar temperaturas de riesgo que podrían afectar de forma negativa al cultivo. Además, una de las aplicaciones en las que este modelo puede tener un impacto mayor es 
en la producción de biomasa, mejorando los modelos de estimación existentes, como el modelo descrito en [2], en el que se mejoraría el comportamiento biólogico de las microalgas y la influencia de la temperatura.

\section{MATERIALES Y MÉTODOS}

En esta sección se presentan el reactor empleado para adquirir datos y contrastar los resultados, así como la descripción del modelo basado en el balance térmico.

\subsection{Reactor abierto}

El reactor empleado para el modelado se localiza en la "Estación Experimental Las Palmerillas" perteneciente a la Fundación Cajamar en Almería, España. Este reactor puede apreciarse en la figura 3 $\mathrm{y}$ tiene una superficie total de $100 \mathrm{~m}^{2}$, formado por dos canales de $50 \mathrm{~m}$ conectados por unas bandas en forma de $\mathrm{U}$ de $1 \mathrm{~m}$ de ancho. El reactor se opera a una profundidad constante de $0.2 \mathrm{~m}$ o menos $\left(20 \mathrm{~m}^{3}\right.$ de volumen máximo total), como se recomienda en [8] para alcanzar el máximo rendimiento hidraúlico en términos de consumo energético. La mezcla se realiza con una rueda de paletas de $1.2 \mathrm{~m}$ de diámetro con ocho palas de contrachapado marino, operada mediante un motor eléctrico. Por otro lado, el proceso de carbonatación se lleva a cabo en un sumidero (1 $\mathrm{m}$ de profundidad y $0.65 \mathrm{~m}$ de ancho) a $1.8 \mathrm{~m} \mathrm{de}$ la rueda de palas. Tres difusores de membrana en el fondo inyectan $\mathrm{CO}_{2}$ y aire en el sumidero.

La cepa de microalga utilizada en este caso se corresponde a Scenedesmus almeriensis (CCAP 276/24). Este tipo de microalgas se caracteriza por una alta velocidad de crecimiento, soportando temperaturas de hasta $45{ }^{\circ} \mathrm{C}$ y valores de $\mathrm{pH}$ que van desde 7 hasta 10. Las condiciones óptimas de crecimiento son $30{ }^{\circ} \mathrm{C}$ y un valor de $\mathrm{pH}$ alrededor de 8 .

\subsection{Balance térmico}

El balance térmico descrito en este artículo se basa en primeros principios y fórmulas empíricas definidas para la transferencia de energía debida a la irradiancia solar, la radiación de onda larga, la evaporación, la convección y la conducción.

Tomando como base el modelo descrito en [12], se han comprobado y establecido los balances de energía que afectan de forma determinante al medio de cultivo y se ha desarrollado un nuevo balance que permita estimar la temperatura del medio en que se desarrolla el cultivo a partir de variables fácilmente medibles. La entrada de radiación solar procede de datos medidos por un sensor de radiación. La radiación de onda larga se calcula mediante la expresión de Stefan-Boltzmann

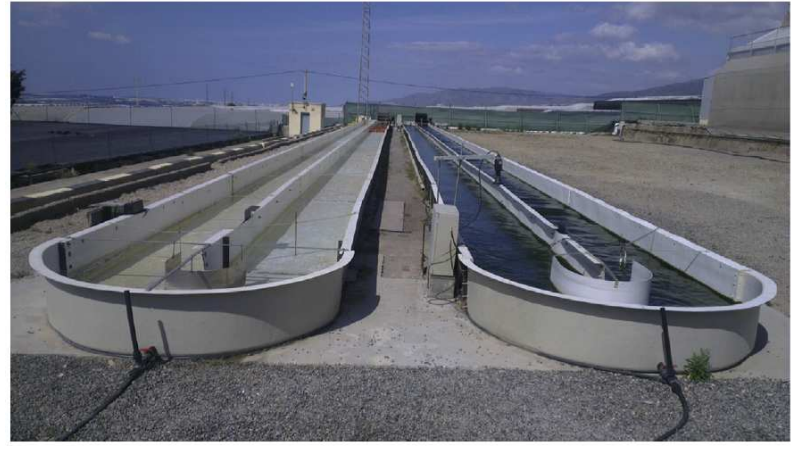

Figura 1: a) Reactor abierto.

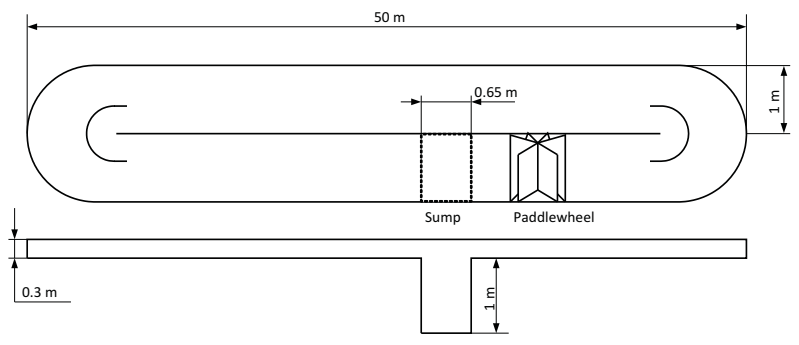

Figura 2: b) Esquema del reactor.

Figura 3: Reactor abierto empleado para el modelado.

[3]. La información respecto al flujo de evaporación es variada en la literatura [1] y su cálculo no es sencillo [11]. En este caso, el balance de energía por evaporación se calcula a partir de los coeficientes de intercambio de calor introducidos por McMillan [7]. La convección se expresa mediante la Ley de enfriamiento de Newton. Por último, la conducción se expresa como la transferencia de calor entre la masa de agua del reactor y la capa de fibra de vidrio que aísla el reactor del suelo. Como resultado del cálculo de los balances energéticos introducidos, el balance térmico se expresa a partir de la siguiente ecuación:

$$
\begin{aligned}
Q_{\text {Total }} & =Q_{\text {irradiancia }}+Q_{\text {radiación }}+Q_{\text {evaporación }}+ \\
& +Q_{\text {convección }}+Q_{\text {conducción }}-Q_{\text {acumulado }}
\end{aligned}
$$

dónde $Q_{\text {Total }}(W)$ representa el resultado del balance térmico, $Q_{\text {acumulado }}(W)$ es el calor acumulado en el reactor, $Q_{\text {irradiancia }}(W)$ representa el flujo de calor a partir de la luz solar, $Q_{\text {radiación }}(W)$ es el flujo de calor a partir de radiación de onda-larga, $Q_{\text {evaporación }}(W)$ muestra el flujo de calor producido por el proceso de evaporación, $Q_{\text {convección }}(W)$ es el flujo de calor causado por convección y $Q_{\text {conducción }}(W)$ representa el flujo de calor entre el reactor y la fibra de vidrio bajo el mismo a través del proceso de conducción.

\subsubsection{Flujo de calor acumulado}

El calor acumulado en el reactor representa la suma de todos los términos de energía que afectan al reactor. 
Viene representado por la siguiente ecuación:

$$
Q_{\text {acumulado }}=V \cdot C_{p} \cdot \rho \cdot \frac{d T_{w}}{d t}
$$

con $V\left(\mathrm{~m}^{3}\right)$ el volumen del reactor, $C_{p}\left(\mathrm{~J} \mathrm{~kg}^{-1}{ }^{\circ} \mathrm{C}^{-1}\right)$ la capacidad calorífica del medio de cultivo, $\rho\left(\mathrm{kg} \mathrm{m}^{-3}\right)$ la densidad del medio y $T_{w}\left({ }^{\circ} \mathrm{C}\right)$ la temperatura del medio en el reactor.

\subsubsection{Flujo de calor por efecto de la irradiancia solar}

El flujo de calor debido a la irradiancia solar incidente sobre la superficie del reactor representa la principal entrada de calor en el reactor. Viene expresado por la siguiente ecuación:

$$
Q_{\text {irradiancia }}=I_{g} \cdot a \cdot A
$$

donde $I_{g}\left(W m^{-2}\right)$ es la radiación solar global, $a(-)$ es la absortividad y $A\left(\mathrm{~m}^{2}\right)$ representa la superficie total del reactor.

\subsubsection{Pérdidas de calor por radiación}

El reactor emite energía térmica como radiación de onda larga. El flujo de energía radiada entre el reactor y el cielo se calcula mediante la siguiente ecuación:

$$
Q_{\text {radiación }}=\sigma \cdot A \cdot e \cdot\left(T_{\text {sky }}{ }^{4}-\left(T_{w}+273,15\right)^{4}\right)
$$

con $\sigma \quad\left(\begin{array}{lll}W & m^{-2} & K^{-4}\end{array}\right) \quad$ la constante de Stefan-Boltzmann, $e(-)$ la emisividad del agua y $T_{s k y}(K)$ la temperatura equivalente del cielo, expresada en [3] con la siguiente fórmula:

$$
\begin{array}{r}
T_{\text {sky }}=\left(273,15+T_{\text {amb }}\right)\left(0,711+0,0056 \cdot T_{\text {dew }}\right. \\
\left.\cdot 0,000073 \cdot T_{\text {dew }}^{2}+0,13 \cdot \cos 15 \cdot t_{\text {solar }}\right)^{0,25}
\end{array}
$$

siendo $T_{a m b}\left({ }^{\circ} \mathrm{C}\right)$ la temperatura ambiente, $T_{\text {dew }}\left({ }^{\circ} \mathrm{C}\right)$ la temperatura de rocío y $t_{\text {solar }}$ representa el número de horas después de la medianoche.

\subsubsection{Flujo de calor por evaporación}

El proceso de evaporación representa la principal fuente de pérdida de calor en el reactor y depende de la forma del reactor, la velocidad del viento y el movimiento del agua, tal como se muestra en [11]. Este balance térmico entre la masa de agua y el ambiente puede establecerse como la diferencia en las presiones de vapor entre el aire del ambiente y la masa de agua del reactor o, de otra manera, teniendo en cuenta la masa de agua evaporada a lo largo del tiempo. Para el cálculo del mismo en el modelo se ha tenido en cuenta el primer método, utilizando para ello la presiónes del agua y del aire. La siguiente ecuación representa el balance por evaporación:

$$
Q_{\text {evaporación }}=A \cdot h_{\text {evap }} \cdot\left(p_{S}^{\prime}-p_{A}^{\prime}\right)
$$

donde $h_{\text {evap }}\left(\begin{array}{lll}W & \mathrm{~m}^{-2} & \mathrm{~Pa}^{-1}\end{array}\right)$ es un coeficiente de intercambio por evaporación, $p_{S}^{\prime}$ es la presión saturada del agua a la temperatura del medio y $p_{A}^{\prime}$ es la presión de vapor del aire a temperatura ambiente. El coeficiente de intercambio se puede calcular usando una expresión empírica, dependiente de la velocidad del viento, presentada en [7].

$$
h_{\text {evap }}=0,036+0,025 \cdot v
$$

siendo $v\left(m s^{-1}\right)$ la velocidad del viento.

Para el cálculo de las presiones de vapor del medio $p_{S}^{\prime}$ y del ambiente $p_{A}^{\prime}$ se ha hecho uso de la ecuación de Antoine aplicada al agua:

$$
p^{\prime}=10^{\left(8,07131-\frac{1730,63}{(233,426+T)}\right)}
$$

\subsubsection{Flujo de calor por convección}

El fenómeno de convección se produce entre la masa de agua del reactor y el aire en el ambiente, resultando en un balance positivo o negativo dependiendo del momento del día y la temperatura ambiente. El balance por convección viene representado por la siguiente ecuación:

$$
Q_{\text {convección }}=h_{\text {conv }} \cdot A \cdot\left(T_{a m b}-T_{w}\right)
$$

con $h_{\text {conv }}\left(W \mathrm{~m}^{-2}{ }^{\circ} \mathrm{C}^{-1}\right)$ siendo el coeficiente de transferencia por convección, $T_{a m b}\left({ }^{\circ} \mathrm{C}\right)$ la temperatura ambiente y $T_{w}\left({ }^{\circ} \mathrm{C}\right)$ la temperatura del medio en el reactor.

\subsubsection{Flujo de calor por conducción}

El balance térmico de conducción representa el intercambio térmico entre el reactor y la superficie bajo el mismo. El material aplicado para la construcción del fondo del reactor ha sido fibra de vidrio. La siguiente ecuación representa el balance por conducción:

$$
Q_{\text {conducción }}=K_{\text {soil }} \cdot x_{\text {soil }} \cdot A_{\text {soil }} \cdot\left(T_{\text {soil }}-T_{w}\right)
$$

donde $K_{\text {soil }}\left(W \mathrm{~m}^{-3}{ }^{\circ} \mathrm{C}^{-1}\right)$ es el coeficiente de transferencia por conducción para la fibra de vidrio, $x_{\text {soil }}(m)$ representa el espesor de la capa de fibra de vidrio inferior del reactor, $A_{\text {soil }}\left(\mathrm{m}^{2}\right)$ es la superficie del reactor en contacto con el suelo, $T_{\text {soil }}\left({ }^{\circ} \mathrm{C}\right)$ representa la temperatura de la fibra de vidrio bajo el reactor y $T_{w}\left({ }^{\circ} \mathrm{C}\right)$ la temperatura del medio en el reactor. 


\subsection{Parámetros}

El valor de los parámetros utilizados en este estudio se encuentran en el cuadro 1.

Cuadro 1: Parámetros y constantes del modelo

\begin{tabular}{|c|c|c|c|}
\hline Parámetro & Descripción & Valor & Unidad \\
\hline V & Volumen de medio en el reactor & 17,2352 & {$\left[\mathrm{~m}^{3}\right]$} \\
\hline$C_{p}$ & Capacidad calorífica del medio & 4184 & {$\left[\mathrm{~J} \mathrm{~kg}^{-1}{ }^{\circ} \mathrm{C}^{-1}\right]$} \\
\hline$\rho$ & Densidad del medio & 1000 & {$\left[\mathrm{~kg} \mathrm{~m}^{-3}\right]$} \\
\hline$a$ & Absortividad & 0,7 & {$[-]$} \\
\hline$A$ & Superficie total del reactor & 100,9752 & {$\left[\mathrm{~m}^{2}\right]$} \\
\hline$\sigma$ & Constante de Stefan-Boltzmann & $5,6697 \cdot 10^{-8}$ & {$\left[\mathrm{~W} \mathrm{~m}^{-2} \mathrm{~K}^{-4}\right]$} \\
\hline$e$ & Emisividad del agua & 0,91 & {$[-]$} \\
\hline$T_{\text {dew }}$ & Temperatura de rocío & 14 & {$\left[{ }^{\circ} \mathrm{C}\right]$} \\
\hline$h_{\text {conv }}$ & Coeficiente de convección & 32,6080 & {$\left[\mathrm{~W} \mathrm{~m}^{-2}{ }^{\circ} \mathrm{C}^{-1}\right]$} \\
\hline$K_{\text {soil }}$ & Coeficiente de conducción (fibra de vidrio) & 0,04 & {$\left[\mathrm{~W} \mathrm{~m}^{-3}{ }^{\circ} \mathrm{C}^{-1}\right]$} \\
\hline$x_{\text {soil }}$ & Espesor de la fibra de vidrio & 0,0017 & {$[m]$} \\
\hline$A_{\text {soil }}$ & Superficie bajo el área del reactor & 100,9752 & {$\left[m^{2}\right]$} \\
\hline
\end{tabular}

\section{RESULTADOS Y DISCUSIÓN}

Esta sección presenta los resultados obtenidos del modelo de temperatura en cuatro partes: una primera parte donde se muestran los resultados de los balances térmicos a partir de datos reales, una segunda parte donde se muestran los resultados respecto a la temperatura estimada por el modelo, una tercera parte donde se describe el método de calibración empleado en el modelo de temperatura y los parámetros calibrados; y por último, una cuarta parte donde se realiza una discusión sobre los resultados obtenidos.

\subsection{Modelo de balances térmicos}

La figura 4 muestra los valores de cada balance térmico para un periodo de tres días utilizando datos reales de temperatura. Las variables reales medidas para el cálculo de los balances de energía se muestran en la figura 5. Observando la figura 4, en azul (a - f) se muestran los términos de potencia que intervienen en el balance global, mientras que la gráfica roja (g) representra el resultado del balance expresado en la ecuación (1). Como se puede observar en la primera gráfica (4.a) de la imagen, el flujo de calor por irradiancia supone la entrada de calor principal en el reactor y depende de la radiación solar, la cual puede apreciarse en la figura 5. La gráfica 4.b de la imagen representa las pérdidas de calor por radiación, las cuales reducen el calor acumulado en el reactor y dependen de la diferencia de temperatura entre el reactor y el cielo. La gráfica 4.c representa el flujo de calor por evaporación, que depende de la diferencia entre las presiones de vapor del agua y del ambiente. El valor de este flujo puede ser positivo por el proceso de condensación nocturna o negativo por el proceso de evaporación. El flujo de calor por convección se muestra en la gráfica $4 . d$ y depende de la diferencia de la temperatura del reactor y el ambiente. Tal como se puede apreciar, este flujo de calor es positivo durante el periodo diurno en el que gana calor del aire y negativo durante el periodo nocturno en el que el reactor cede calor al ambiente por el proceso de convección. La gráfica 4.e presenta el calor por conducción, que depende de la diferencia entre la temperatura del reactor y la temperatura bajo su superficie. El suelo actúa como una capacitancia térmica dónde se almacena calor durante el periodo diurno (cedido por el reactor) y que cede calor durante el periodo nocturno. Se ha considerado una temperatura del suelo constante equivalente a la media obtenida durante el día completo.

El calor acumulado presentado en la gráfica 4.f puede calcularse a partir de la diferencia de temperatura del reactor en función del tiempo y representa la suma de todos los balances térmicos anteriores. El balance térmico en el reactor es un proceso en equilibrio por lo que el resultado del mismo debe ser de media nula, representado por el flujo de calor total $\left(Q_{\text {Total }}\right)$ en la gráfica $4 . g$, en rojo, donde se muestran además, la contribución de todos los flujos anteriores. El flujo de calor total representa un indicador en la precisión del modelo, ya que la variación media del mismo debe dar un resultado cercano a cero. Para el balance de tres días representado en la figura 4 , se obtuvo un resultado de flujo de calor total medio de $\bar{Q}_{\text {Total }}=-0,28$. Este resultado indica que los balances establecidos en la sección 2 son correctos y tienen sentido físico, por lo que es posible estimar la temperatura del reactor a partir de un modelo compuesto por los flujos de calor presentados.

\subsection{Modelo de temperatura}

La evolución dinámica de la temperatura se obtiene a partir de la ecuación (1) en el equilibrio, basada en los balances térmicos descritos y reformulada como la siguiente ecuación:

$$
\begin{aligned}
& V \cdot C_{p} \cdot \rho \cdot \frac{d T_{w}}{d t}=Q_{\text {irradiancia }}+Q_{\text {radiación }}+ \\
& +Q_{\text {evaporación }}+Q_{\text {convección }}+Q_{\text {conducción }}
\end{aligned}
$$

A partir de unas condiciones iniciales de temperatura, es posible estimar la temperatura del reactor a lo largo del tiempo a partir de la ecuación propuesta.

La figura 6 muestra una comparación entre la temperatura real (segmentada azul) y la temperatura estimada por el modelo (rojo) para dos casos de estudio: un periodo de tres días (figura 6.a), utilizado para calibración, que corresponde al balance térmico mostrado en la sección $3.1 \mathrm{y}$, por otro lado, un ensayo de validación que corresponde a un periodo de dos días consecutivos (figura 6.b). En la figura figura 6.a se aprecia como la temperatura estimada sigue la misma tendencia que la temperatura real, con un error máximo de $M A X_{\text {err }}=1,66$, las cuales son tolerables para el estudio en el comportamiento 

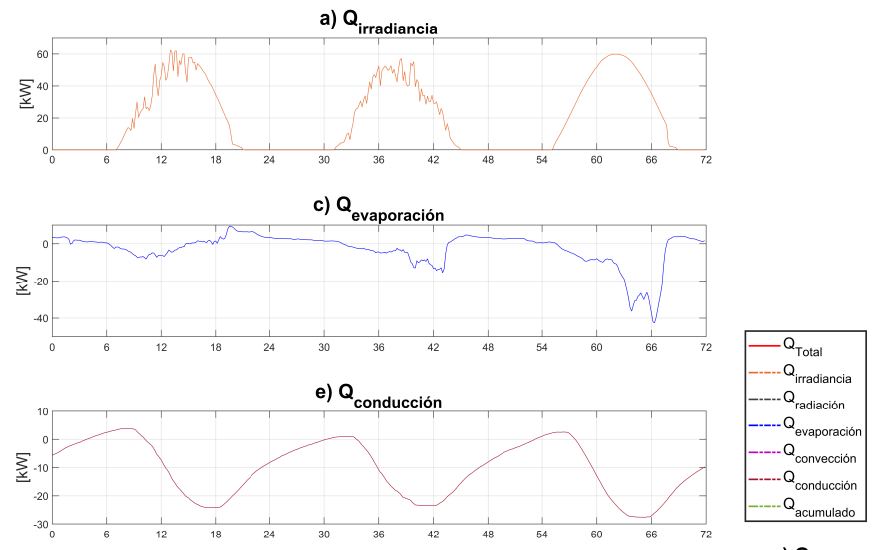

g) $Q_{\text {Total }}$
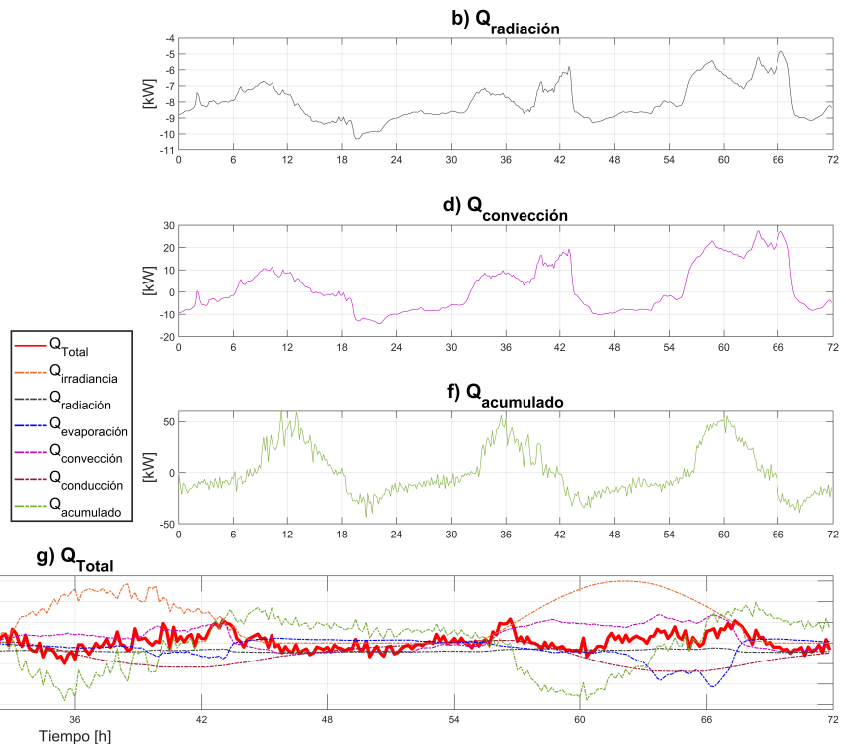

Figura 4: Balances térmicos individuales y balance total de calor (rojo).
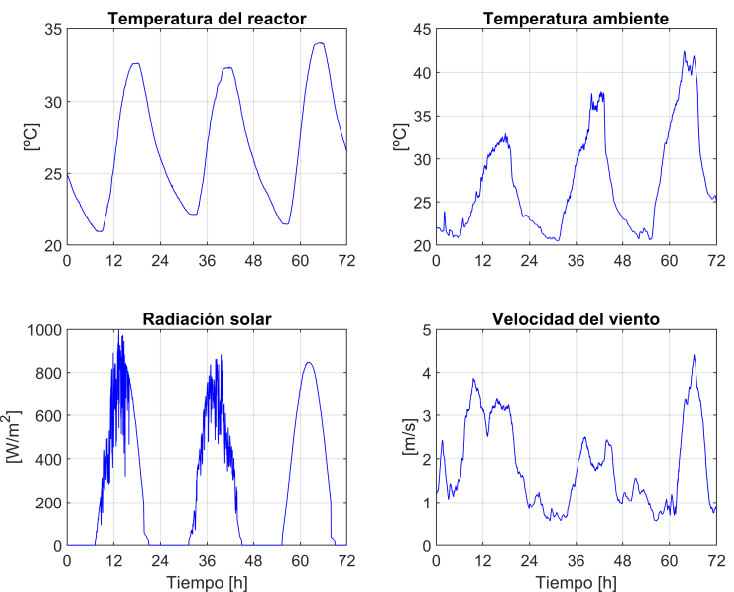

Figura 5: Variables medidas del sistema

de las microalgas. Como índice de precisión se ha empleado el error cuadrático medio entre las dos curvas, obteniéndose un valor de $R M S E=0,67$.

En el caso de validación presentado en la figura 6.b, se muestra otro ensayo de temperatura estimada para dos días, en el que se aprecia un ligero desajuste en el primer día respecto al segundo. Para este caso, el error cuadrático medio es de $R M S E=0,70$, mientras que el error máximo obtenido es de $M A X_{\text {err }}=1,88$, un valor aceptable y dentro de los límites de tolerancia aplicados.

\subsection{Calibración}

Las ecuaciones desarrolladas en la sección 2 hacen uso de una serie de parámetros cuyo valor exacto se desconoce $\mathrm{o}$, en su defecto, se conoce su valor en un rango definido. La incertidumbre en el valor de estos parámetros pone de manifiesto la necesidad un proceso de calibración, el cual se ha realizado mediante algoritmos genéticos. La calibración mediante algoritmos genéticos resulta en un método útil y fiable en la estimación de parámetros inciertos, ya que permite optimizar la función de coste cuadrática que mide la desviación de la salida del modelo y la del sistema real modificando los valores de los parámetros entre los límites establecidos. Los datos del periodo de tres días mostrados en el primer apartado de esta sección se han empleado junto con el algoritmo genético para estimar los valores del coeficiente de convección $\left(h_{\text {conv }}\right)$, el coeficiente de conducción $\left(K_{\text {soil }}\right)$ y el espesor de la capa de fibra de vidrio inferior del reactor $\left(x_{\text {soil }}\right)$. El coeficiente de convección es un parámetro muy característico cuyo valor se ha determinado en un rango de 4 a 80 [ kcal $h^{-1} \mathrm{~m}^{-2}{ }^{\circ} \mathrm{C}^{-1}$ ]. El coeficiente de conducción para la fibra de vidrio tiene un rango de 0.03 hasta $0.07\left[\mathrm{~W} \mathrm{~m}^{-3}{ }^{\circ} \mathrm{C}^{-1}\right]$, mientras que el espesor del mismo oscila entre 1 y $2[\mathrm{~mm}]$. El rango de los parámetros estimados se ha obtenido de la literatura citada, así como de la propia experiencia en el diseño de la instalación.

\subsection{Discusión}

El error obtenido en los ensayos de la figura 6 denota una precisión aceptable en el modelo obtenido a partir de los balances térmicos. Desde el punto de vista biológico de las microalgas, una variación de un grado respecto a la temperatura estimada y la real no supondría problema alguno en la representación de la dinámica de las mismas. La gráfica de la izquierda en la figura 6 presenta un desajuste durante 


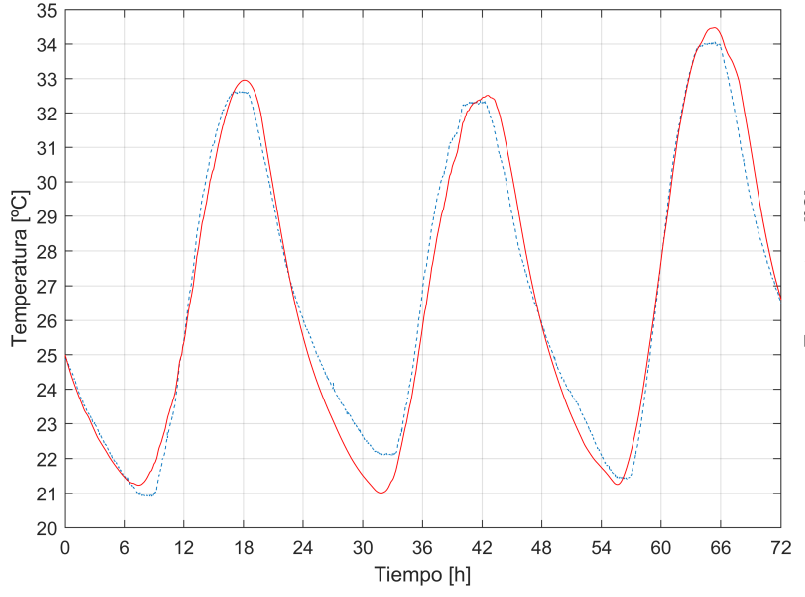

a) Datos de calibración.

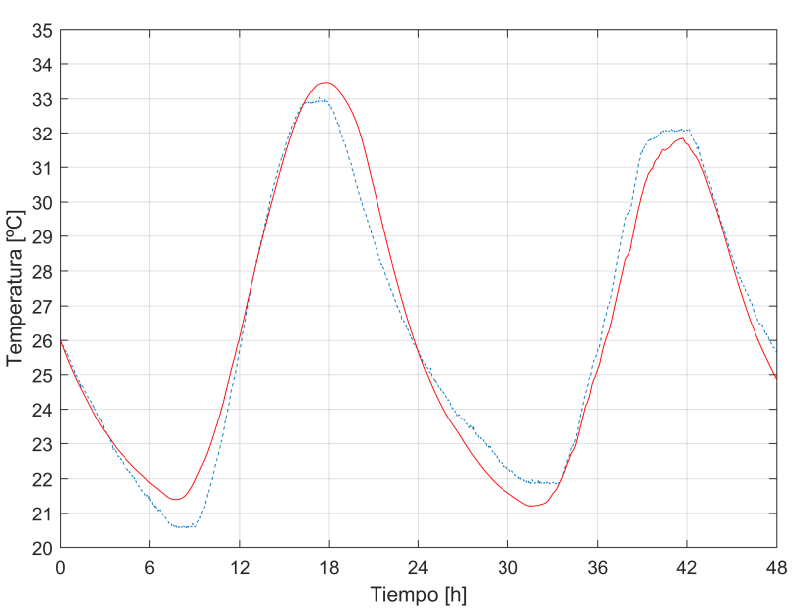

b) Datos de validación.

Figura 6: Temperatura real (azul) vs temperatura estimada (rojo).

el periodo nocturno del segundo día, debido a una infraestimación de la temperatura, al contrario que el desajuste en la gráfica de la derecha, dónde se produce una sobrestimación de la temperatura durante el periodo nocturno del primer día, que tiene efectos durante el periodo diurno del mismo. Estos desajustes pueden deberse a la existencia de perturbaciones no medibles y que no se han contemplado en los balances térmicos. Por otro lado, la calibración mediante algoritmos genéticos permite obtener el valor de aquellos parámetros que presentan una incertidumbre en la influencia sobre la temperatura del reactor. Los resultados globales obtenidos han sido positivos y destacables para el uso del modelo en el desarrollo de aplicaciones como la combinación del mismo con modelos de microalgas donde se analiza su dinámica y otros parámetros como productividad, rendimiento, consumo de $\mathrm{CO}_{2}$ y evolución de $\mathrm{pH}$.

\section{CONCLUSIONES}

Este trabajo presenta un modelo de temperatura para reactores abiertos basado en un balance térmico a partir de las condiciones medibles en el ambiente. Los resultados de la evolución dinámica de la temperatura obtenidos a partir del modelo muestran unos valores satisfactorios que se asemejan en gran medida a los valores de temperatura reales medidos en el reactor. Se ha demostrado en la literatura el gran impacto de la temperatura en la productividad de las microalgas $\mathrm{y}$, es por ello, que este tipo de modelos tienen un papel fundamental en el desarrollo de nuevos y más completos modelos de microalgas que permitan comprender en profundidad todos los parámetros que afectan a su crecimiento. El uso de modelos en los que se tengan en cuenta todas las variables que afectan a las microalgas es escaso en la práctica, por tanto, este modelo de temperatura tiene como objetivo complementar el uso de modelos más completos que permitan desarrollar aplicaciones de evaluación precisas en el campo de las microalgas, como control óptimo de reactores, estudios de impacto de variables, mejora de rendimiento o estimación de parámetros. En futuros trabajos se analizará la presencia de perturbaciones que pudieran afectar a los balances de flujos de calor, como por ejemplo, las pérdidas por evaporación.

\section{Agradecimientos}

Este trabajo ha sido financiado con el Proyecto del Plan Nacional DPI2017-84259-C2-1-R del Ministerio de Economía y Competitividad y Fondos FEDER, y el programa European Union's Horizon 2020 Research and Innovation Program bajo el proyecto SABANA 727874 .

\section{English summary \\ TEMPERATURE MODEL FOR A MICROALGAE RACEWAY REACTOR}

\begin{abstract}
This paper presents a temperature model for raceway reactors based on a thermal balance from easily measurable variables of the environment. The model allows to estimate the temperature with precision, following the dynamics observed in the temperature of the reactor studied. Thanks to this type of models, more complete microalgae models can be developed, thus faithfully representing its growth dynamics.
\end{abstract}

Keywords: Temperature model, microalgae, raceway reactor. 


\section{Referencias}

[1] Bechet Q., Shilton A., Park J.B.K., Craggs R.J., Guieysse B. (2011). Universal temperature model for shallow algal ponds provides improved accuracy, Environmental Science and Technology, 45: 3702-3709.

[2] Costache T.A., Acién F.G., Morales M.M., Fernández-Sevilla J.M., Stamatin I., Molina E. (2013). Comprehensive model of microalgae photosynthesis rate as a function of culture conditions in photobioreactors, Applied Microbiology and Biotechnology, 97(17): 7627-7637.

[3] Duffie J.A., Beckman W.A. (1991). Solar Engineering of Thermal Processes, 2nd ed. Wiley-Interscience, Toronto.

[4] Hempel N., Petrick I., Behrendt F. (2012). Biomass productivity and productivity of fatty acids and amino acids of microalgae strains as key characteristics of suitability for biodiesel production, Journal of Applied Phycology, 24: 1407-1418.

[5] James S.C., Boriah V. (2010). Modeling algae growth in an open-channel raceway, Journal of Computational Biology, 17: 895-906.

[6] Marsullo M., Mian A., Ensinas A.V., Manente G., Lazzaretto A., Marechal F. (2015). Dynamic modeling of the microalgae cultivation phase for energy production in open raceway ponds and flat panel photobioreactors, Frontiers in Energy Research, 3: 41-59.

[7] McMillan W. (1971). Heat Dispersal - Lake Trawsfynfydd Cooling Studies, Symposium on Freshwater Biology and Electrical Power Generation, 1: 41-80.

[8] Mendoza, J.L., Granados, M.R., de Godos, I., Acién, F.G., Molina, E., Banks, C.J., Heaven S. (2013). Fluid-dynamic characterization of real-scale raceway reactors for microalgae production, Biomass Bioenergy, 54: 267-275.

[9] Pawloski A., Mendoza J.L., Guzmán J.L., Berenguel M., Acién F.G., Dormido S. (2015). Selective pH and dissolved oxygen control strategy for a raceway reactor within an event-based approach, Control Engineering Practice, 44: 209-218.

[10] Rodolfi L. Chini Zittelli G., Bassi N., Padovani G., Biondi N., Bonini G., Tredici M.R. (2009). Microalgae for oil: strain selection, induction of lipid synthesis and outdoor mass cultivation in a low-cost photobioreactor, Biotechnology and Bioengineering, 102: $100-112$.

[11] Sartori E. (2000). A critical review on equations employed for the calculation of the evaporation rate from free water surfaces, Solar Energy, 68: 77-89.

[12] Slegers P.M., Lösing M.B., Wijffels R.H., van Straten G., van Boxtel A.J.B. (2013). Scenario evaluation of open pond microalgae production, Algal Research, 2: 358-368.

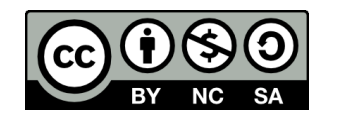
(C) 2019 by the authors.
Submitted for possible
open access publication under the terms and conditions of the Creative Commons Attribution CC BY-NC-SA 4.0 license (https://creativecommons.org/licenses/by-nc-sa/4.0/deed.es). 